\title{
Yttrium-90 (Y-90) Resin Microsphere Therapy for Patients with Unresectable Hepatocellular Carcinoma: a Single-Center Experience
}

\author{
Semra İnce ${ }^{1}$ • Bülent Karaman ${ }^{2}$ • Engin Alagoz ${ }^{1}$ - Nuri Karadurmuş ${ }^{3}$ Hüseyin Şan ${ }^{1}$. \\ Cemal Nuri Erçin ${ }^{4} \cdot$ Nuri Arslan ${ }^{1}$
}

Published online: 20 June 2017

(C) Springer Science+Business Media New York 2017

\begin{abstract}
Background/Aim Selective intraarterial radionuclide therapy (SIRT) with yttrium-90 (Y-90) resin microspheres presently has successful results in primary or metastatic inoperable liver tumors. This procedure, which is also known as radioembolisation, delivers high doses of radiation selectively to hepatic tumors while minimum healthy liver exposure. The aim of this study was to present our clinical experience of radiomicrosphere therapy for the treatment of patients with unresectable hepatocellular carcinoma (HCC).

Methods We performed 40 Y-90 microsphere therapies in 28 patients (5 females, 23 males; mean age \pm SD $48 \pm 8$ ) with HCC during the period from April 2008 through December 2016. Pretreatment Tc-99m microaggregated albumin (MAA) scintigraphy was performed to all patients in order to detect eligibility for SIRT. All patients had pre- and post-biochemical tests (hemogram and serologic tests) and imaging methods (CT or MRI or PET/CT) at regular intervals to detect any possible complication and determine response rates.
\end{abstract}

Semra İnce

drsemra@gmail.com

1 Department of Nuclear Medicine, Gulhane Training and Research Hospital, Etlik, Ankara, Turkey

2 Department of Radiology, Gulhane Training and Research Hospital, Etlik, Ankara, Turkey

3 Department of Medical Oncology, Gulhane Training and Research Hospital, Etlik, Ankara, Turkey

4 Department of Gastroenterology, Gulhane Training and Research Hospital, Etlik, Ankara, Turkey
Results The mean shunting to the lungs on MAA scan was $6.5 \%$ and the mean \pm SD administered dose of Y-90 was $1.55 \pm 0.32 \mathrm{GBq}$ in all patients. The estimated doses to the target tumors, normal liver parenchyma and lungs were $105.7 \pm 55.3,25.5 \pm 8.2$ and $5.8 \pm 1.7 \mathrm{~Gy}$, respectively. No significant complication was observed during or early after (first week) the treatment procedure and it was well tolerated by all the patients. Only one patient developed a treatment-related gastroduodenal ulcer 3 weeks after the treatment. In control imaging tests (MRI or FDG PET/CT) performed 2.5 months after the treatment, we observed complete response in $2(7 \%)$ patients, partial response in $10(36 \%)$ patients, stable disease in $5(18 \%)$ patients and progressive disease in $11(39 \%)$ patients.

Conclusion According to our clinical experience, we can conclude that Y-90 microsphere therapy is a safe and effective treatment option for the patients with unresectable HCC without any serious side effects. 